\title{
LA ARTICULACIÓN TERRITORIAL DE GALICIA CON EL RESTO DEL ESTADO. APORTACIONES DE LA CONECTIVIDAD AL DESARROLLO REGIONAL
}

José Antonio DÍAZ FERNÁNDEZ

Departamento de Geografia, Universidad de Santiago

El presente trabajo centra todos sus propósitos en someter a un examen detenido la situación en la que se encuentra la articulación de Galicia respecto del conjunto de España. En este contexto, nos ocuparemos de la secuencia histórica que siguió la dotación de inversiones públicas por parte de la Administración Central para contribuir al desarrollo regional de Galicia. Será en este mismo ámbito donde mejor se apreciarán los constantes retrasos a los que estuvo sometido el desarrollo regional gallego, hasta un punto tal que Galicia experimentó un profundo estancamiento en su desarrollo socioeconómico, tal y como lo demuestran los valores de la TABLA No 1 .

Tabla $n^{0}$ 1. Distribución sectorial en (\%) de la ocupación en Galicia y España para 1960-1970.

\begin{tabular}{ccccr}
\hline Sector & \multicolumn{1}{c}{$\mathbf{1 9 6 0}$} & \multicolumn{2}{c}{$\mathbf{1 9 7 0}$} \\
\hline & Galicia & España & Galicia & España \\
\hline Agricultura & 65,4 & 41,7 & 57,8 & 32,1 \\
Industria & 18,4 & 31,7 & 19,5 & 33,9 \\
Servicios & 16,2 & 26,6 & 22,5 & 33,7 \\
\hline \multicolumn{2}{l}{ FUENTE: } & Dirección General de Empleo. Ministerio de Trabajo. Banco Bilbao: Dis- \\
& tribución de la renta provincial. Elaboración Propia.
\end{tabular}

A título de ejemplo de lo advertido, expondremos, en primer lugar, el acusado lastre que la emigración hacia el exterior supuso, para después ratificar dicha aseveración mediante la escasa transformación que su tejido socioproductivo había conocido en el período 1960-1970, ya que el peso del sector primario era considerablemente más importante que el de la media nacional. Aún más, cuando en los decenios de los 60 y 70 en el conjunto de España se estaba produciendo una intensa fase de industrialización y terciarización de la economía, Galicia permanecía anclada en una estructura productiva escasamente diversificada, dotada de un bajo nivel de capitalización y, por tanto, poco competitiva. Así pues, visto desde esta perspectiva, el escaso empuje del desarrollo gallego dio pie a que esta región recibiese el no menos justificado apelativo de Periferia Atlántica. 
Múltiples son las claves explicativas que se apuntan para justificar la escasa capacidad de transformación social y económica de Galicia dentro del conjunto del Estado. Nuestro propósito es detenernos en una de las mismas que, de alguna forma, participa y explica la existencia de las demás como es la dotación de infraestructuras de transportes y comunicaciones y el papel que éstas cumplen dentro de la dinamización capilar del tejido productivo de Galicia. Cierto es que la insuficiencia continuada de inversión pública por parte del Estado en materia de comunicaciones y transportes, tiende a penalizar la igualdad de oportunidades y a condicionar, en paralelo, la necesidad de transformación económica y social.

\section{LA ARTICULACIÓN TERRITORIAL COMO BASE DEL DESA- RROLLO REGIONAL.}

Expuesto de este modo y por extensión de lo anterior, la ausencia de inversiones en la modernización de la estructura productiva llevaba aparejada la consiguiente pérdida de competitividad de la economía regional frente a aquellas otras regiones que sí disfrutaban de una mayor dotación de inversiones públicas (SEQUEIROS TIZÓN, X. G., 1979). En consecuencia, estas últimas estarían ubicadas en mejor posición para incidir en el arrastre de la inversión privada.

Siguiendo con este argumento, los debates propiciados durante los años 1970 destacaban las actuaciones que deberían ser acometidas en Galicia para lograr un modelo de desarrollo regional equilibrado y autosostenido (PENA TRAPERO, 1972; MARCO BORDETAS, L, 1977). De hecho, la temática central suscitada por la mayoría de los estudios cuestionaba abiertamente la idoneidad del modelo de política de desarrollo regional propuesta en los Planes de Desarrollo Económico y Social (RICHARDSON, H. W, 1971, TAMAMES, R. 1980). Así, se abogaba por un modelo de desarrollo regional descentralizado, capaz de impulsar económicamente las potencialidades de las regiones españolas; toda vez que los Planes de Desarrollo no fueron capaces de consumar un tipo de crecimiento económico regional favorable a las regiones periféricas (GÓMEZ BARAHONA, 1991). La respuesta a tal propósito -impulso del desarrollo regional descentralizado en Galicia- debería estar sustentado en dos pilares de singular relieve. En primer término, era imprescindible la necesaria dotación de inversión pública en infraestructuras de comunicaciones y transportes en un territorio que se encontraba conectivamente aislado del Estado, para así tratar de fomentar sus ventajas comparativas que acabarían redundando en nuevos estándares de competitividad regional; hecho éste que se debiera haber confirmado con la aprobación del Plan de Accesos a Galicia de 1970. En segundo lugar, los derroteros propuestos en la nueva política económica del Estado pasaban por la previa modernización de la estructura económica del país. En consecuencia, estas medidas debían dirigirse hacia la revitalización de la estructura económica regional gallega para incentivar su competitividad en el acceso a los mercados. En suma, la participación conjunta de las dos directrices expuestas trataba de difundir el desarrollo económico regional por todo el territorio gallego a fin de hacer más atractiva la producción regional a la hora de acceder a los mercados. Además, estas dotaciones de inversión pública pretendían captar mayores cotas de inversión privada para así reforzar la perseguida diversificación de la es- 
tructura productiva regional.

Los modelos de planificación económica del desarrollo regional encontraron su máxima expresión en los tres Planes de Desarrollo Económico y Social aprobados, y que en Galicia tan sólo el primero y el tercero de los mismos recogían previsiones de inversión pública. Ahora bien, sus resultados no tuvieron ni el alcance ni la incidencia inicialmente prevista por el equipo de los redactores. En efecto, el I Plan de Desarrollo Económico y Social 1964-1968 dotaba a Galicia de dos Polos de Desarrollo situados en A Coruña y Vigo. En base a los mismos, se pretendía dinamizar económicamente ambas áreas para que, una vez alcanzado el objetivo, se fomentase la irradiación del crecimiento económico a todo el territorio gallego. En el Tercero se propuso la creación del Polo de Desarrollo de Vilagarcía que finalmente no se llevaría a efecto, aunque resultaba de sumo interés la programación de la Gran Area de Expansión Industrial de Galicia (GAEIG). En cambio, los resultados deducidos de la política de los Polos de Desarrollo (CAMBRE MARIÑO, 1968; TABOADA ARCEO, 1969; RICHARDSON, 1970; SEQUEIROS TIZÓN, 1986) han sido bien exiguos en lo que concierne a generación de inversiones privadas, dotación de empleo, modernización y capitalización empresarial, etc. Para empezar, la elección de las dos áreas urbanas que acogieron a los Polos de Desarrollo de A Coruña y Vigo sobre las que se proyectó la dotación de inversión pública en Galicia provocaron efectos perversos. El primero venía a estar referido a la escasa irradiación del crecimiento económico hacia el interior (Lugo y Ourense), por carecer el territorio regional de una oferta de infraestructuras de transporte y comunicaciones de alta capacidad. El segundo nos muestra que en lugar de alcanzarse una eficiente irradiación del crecimiento económico, lo que realmente se consiguió fue un proceso de crecimiento concentrado en favor de las dos áreas urbanas señaladas. Esta adversa realidad puede contribuir a explicar como en esta época se agravaron todavía más los desequilibrios internos en Galicia, especialmente visibles en los movimientos de población hacia las áreas económicamente más prosperas. Con atención al Polo de Vilagarcía, el planeamiento del III Plan había pretendido impulsar el desarrollo de las cabeceras de comarca para así difundir el desarrollo económico a todo el conjunto regional. Sin embargo, existieron dos elementos que hicieron estériles tales pretensiones. Por una parte, la Administración Central había continuado dotando de inversión pública a la fachada atlántica gallega (Vilagarcía de Arousa) en detrimento de los espacios del interior regional; lo cual contribuía a favorecer un modelo de crecimiento polarizado en favor del litoral. En segundo lugar, las previsiones de la GAEIG no alcanzaron el eco esperado por no dotar a Galicia de una red de accesos capaz de fomentar una oferta de conectividad territorial respecto de su sistema de ciudades.

Como corolario de todo lo referido hasta aquí, los estudiosos del desarrollo regional cayeron en la plena cuenta de que el principal problema que aquejaba a Galicia era el aislamiento o también enclavamiento dentro del contexto regional del Estado. Lejanía que estaba presente medida en términos de accesibilidad territorial o, complementariamente, con la carencia de conectividad territorial fundamentada en una oferta de transporte multimodal. Al tratar alguno de los factores que participan con mayor nitidez en la caída de los niveles de accesibilidad por 
parte de la demanda de transporte entre cuyos extremos de viaje está Galicia, mencionaremos, de entrada, el que le venía dado por la propia distancia topológi$c a$ o real existente entre cada punto de la matriz O-D (Origen-Destino) de transporte. Sin embargo, en un análisis comparativo con otras áreas del Estado de semejante distanciamiento, e incluso mayor (Cataluña, Andalucía), la accesibilidad que en los años 60 y 70 se obtenía para cada una de estas regiones era bastante más elevada que la de Galicia (ARESPACOCHAGA Y FELIPE, J., 1967). La explicación de tal disimetría nos obliga a consignar que la estructura del marco físico por el que discurren los trazados de las principales vías de comunicación que facilitan el acceso de Galicia a la Meseta, se inscriben en un perfil altimétrico con cotas bastante más elevadas que las que deben superar aquellas comunicaciones con dirección a Cataluña o Andalucía. Efectivamente, mientras la conectividad territorial de Madrid respecto de Barcelona discurre por altitudes que en su mayor parte se enmarcan por debajo de una cota altimétrica de $\operatorname{los} 630 \mathrm{~m}$., altitud a la que se encuentra Madrid, en el caso concreto de la accesibilidad a un punto de Galicia, tomemos como ejemplo la ciudad de A Coruña, en más de las 3/4 del trayecto entre ambos núcleos el transporte debe salvar cotas superiores a los $700 \mathrm{~m}$. Pero aún más lejos, como bien se advierte en el perfil comparativo de la FIGURA N ${ }^{\circ}$ 1, la demanda de transporte generado o atraído por Galicia y con uno de sus extremos de viaje en Madrid, tiene que salvar altitudes de más de $1.000 \mathrm{~m}$ en más de 1/4 del itinerario.

Tabla $\mathbf{n}^{0}$ 2. Distribución de la superficie territorial según áreas altimétricas en Galicia (en $\mathrm{km}^{2}$ ).

\begin{tabular}{lrrrrr}
\hline & A Coruña & Lugo & $\begin{array}{r}\text { Ouren- } \\
\text { se }\end{array}$ & $\begin{array}{r}\text { Ponteve- } \\
\text { dra }\end{array}$ & Galicia \\
\hline$<=200 \mathrm{~m}$. & $\mathbf{2 . 7 5 8}$ & $\mathbf{5 7 2}$ & $\mathbf{1 6 0}$ & $\mathbf{1 . 5 2 8}$ & $\mathbf{5 . 0 1 8}$ \\
$2001-600 \mathrm{~m}$. & $\mathbf{5 . 0 3 0}$ & $\mathbf{5 . 9 9 1}$ & $\mathbf{2 . 1 1 7}$ & $\mathbf{2 . 1 5 5}$ & $\mathbf{1 5 . 2 9 3}$ \\
$601-1.000 \mathrm{~m}$. & $\mathbf{1 6 3}$ & $\mathbf{2 . 8 0 7}$ & $\mathbf{3 4 9 8}$ & $\mathbf{8 1 0}$ & $\mathbf{7 . 2 7 8}$ \\
$1.001-2.000 \mathrm{~m}$. & - & $\mathbf{4 8 6}$ & $\mathbf{1 . 4 9 8}$ & $\mathbf{2}$ & $\mathbf{1 . 9 8 6}$ \\
\hline
\end{tabular}

FUENTE: INE. Anuario Estadístico de España. 1994.

Además, al encontrarse ubicadas las principales formaciones montañosas gallegas en el cuadrante oriental y sudoriental de las provincia de Lugo y Ou rense respectivamente, siguiendo una disposición meridiana en Lugo (Serra dos Ancares y Courel), mientras en Ourense el relieve presenta una disposición de NW a SE (Serra de Queixa, San Mamede y Eixe), apreciamos como esta particularidad se traduce tácitamente en dos condicionantes claros. De una parte, la dotación de infraestructuras de comunicaciones y transportes que permitan el acceso a Galicia, tienen la complejidad de superar una marcada compartimentación del relieve que le acaba ocasionando, y he aquí el segundo factor, el adverso efecto barrera a la oferta de transporte regional (DÍAZ FERNÁNDEZ, 1997). Paralelamente, el factor del aislamiento o enclavamiento del territorio debido a la disposición del relieve repercute en las demandas de transporte, que bien teniendo uno de sus extremos 
Figura 1. Perfiles comparativos (altitudes, tiempo) de los trazados Madrid-La Coruña y Madrid-Barcelona.

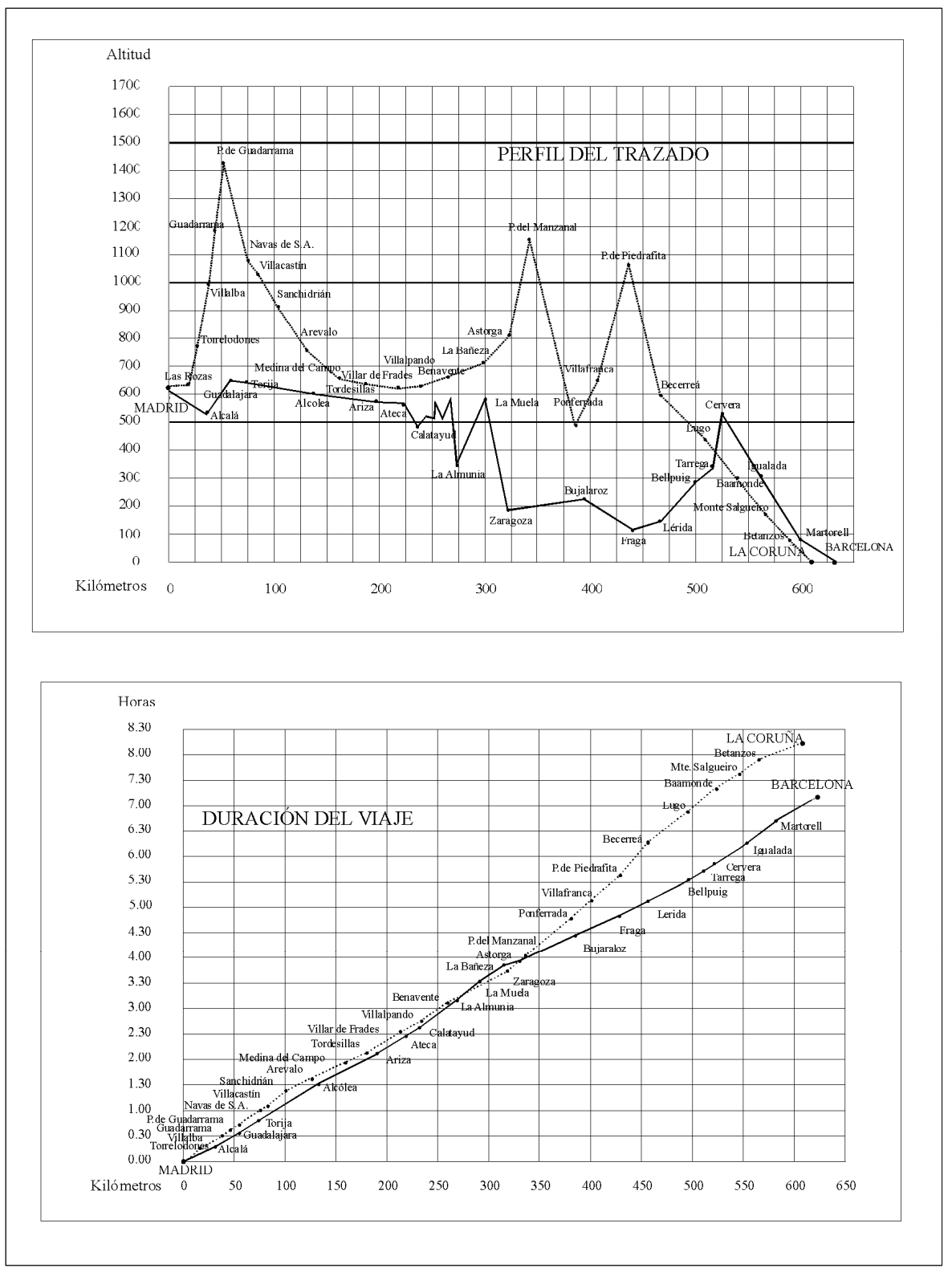


de viaje en Galicia y el otro en Madrid, experimentaban una destacada caída en sus estándares de accesibilidad. Dicho de un modo más simple, los tiempos de viaje alcanzados para las demandas de transporte A Coruña-Madrid eran bastante más amplios que los propiamente alcanzados por Barcelona. Y ello aún cuando esta última ciudad se localizaba a unos $150 \mathrm{~km}$ más de distancia que A Coruña con respecto de Madrid. Así pues, al traducir el nivel de accesibilidad a tiempo de viaje apreciamos como Barcelona distaba una hora y media menos de viaje que $\mathrm{A}$ Coruña.

La razón de tal circunstancia hay que tratar de contemplarla en la caída de las velocidades comerciales de la red regional por causa de las condiciones técnicas del trazado geométrico de la calzada, que es la que incide abiertamente en la capacidad de carga (IMD) de la red viaria regional. Junto a este factor, y relacionado con el mismo, otro hecho que influye en la caída de la velocidad comercial de la red gallega es el elevado índice de sinuosidad de su trazado así como también las pendientes que la red tiene que salvar. Con atención a la incidencia que las características técnicas tienen sobre los niveles de accesibilidad territorial conviene recordar que la mayor o menor fluidez de las demandas de transporte están claramente relacionadas con la anchura de la plataforma, el tipo de firme, los radios de curvatura, el nivel de ondulación del trazado de la red, etc. Así, la interacción de todos estos indicadores junto con el índice de motorización regional confirma como los niveles de demanda diaria de transporte pueden llegar a acusar adversas situaciones de: congestión de tráfico, nivel de saturación de la vía o, por finalizar, la presencia de un considerable deterioro en los estándares de la seguridad vial.

La importancia que sobre la red viaria regional tiene tanto el índice de sinuosidad de la red como el propio perfil de su trazado, le vienen dados a ésta por la disposición meridiana del relieve, así como por las características del poblamiento. En efecto, la dotación de conectividad al hábitat regional gallego se traduce en que el trazado de la red viaria regional debe salvar, previamente, una tupida red hidrográfica; por lo que se hacía preciso dotar a la red regional de ingentes dotaciones presupuestarias destinadas a la construcción de obras de fábrica. Posteriormente, y según la localización de las mayores densidades de población en la fachada litoral, la red regional de comunicaciones terrestres debía seguir un trazado particularmente sinuoso al tener que adaptarse a la morfología de las rías gallegas.

En conclusión, puede afirmarse que la inversión en capacidad de carga de la red (ancho de la plataforma), revierte en el incremento de la accesibilidad territorial, en la mejora de la conectividad regional y, en suma, en la potencialidad que un eje posee para tratar de captar mayores niveles de tráfico futuros toda vez que se logran garantizar unas velocidades comerciales más elevadas. Además de estas ventajas propiciadas por la dotación de infraestructuras de mayor capacidad, estarían los efectos que la red causaría sobre la estructura territorial y económica de Galicia: articulación e integración territorial. Además, la mejora de los ejes de comunicación repercutiría en la consecución de atractivas externalidades positivas propias del sistema de transportes: reducción del índice de siniestrabilidad vial, reducción de costes de transporte (combustible y mantenimiento del parque móvil), gastos por accidentes y reducción de tiempos de viaje. 


\section{LA DEMANDA DE ACCESIBILIDAD Y CONECTIVIDAD TE- RRITORIAL: DOS CLAVES DEL DESARROLLO REGIONAL.}

La comunidad autónoma de Galicia ha visto como su accesibilidad territorial ha atravesado por fases bien diferenciadas dentro del contexto español. En los años 60 y 70, la accesibilidad regional era de las más bajas del conjunto del Estado, actuando este condicionante como un factor añadido más en las insuficiencias del desarrollo regional. El crecimiento de los índices de motorización de Galicia hacían necesario adaptar el nivel de la demanda de transporte existente a una oferta de servicio capaz de garantizar la fluidez del tráfico. Efectivamente, según se advierte en la TABLA $\mathrm{N}^{\circ} 3$, la evolución del nivel de motorización regional ha experimentado una tendencia al crecimiento salvo en el parque de autobuses. Sin embargo, si evaluamos la participación total de su parque móvil respecto a la del conjunto del Estado, apreciamos como ésta es todavía inferior, en términos porcentuales, a la participación poblacional gallega dentro del territorio nacional. En conclusión, esta evidencia nos permite deducir que la tasa de motorización regional todavía puede continuar creciendo, aunque es bien cierto que internamente Galicia reflejará niveles de motorización bastante diferenciados para cada una de sus provincias.

Tabla $\mathbf{n}^{0}$ 3. Evolución del parque regional de vehículos según su tipología. 1991-1994.

\begin{tabular}{lrrrr}
\hline Tipo de vehículo & $\mathbf{1 9 9 1}$ & $\mathbf{1 9 9 3}$ & $\mathbf{1 9 9 4}$ & $\mathbf{\% G / E}$ \\
\hline Turismos & 750.856 & 903.027 & 923.632 & 6,7 \\
Camiones & 124.050 & 151.775 & 155.836 & 5,5 \\
Autobuses & 4.147 & 4.288 & 4.273 & 9,1 \\
Otros & 50.582 & 68.919 & 70.828 & 4,4 \\
\hline Total & 929.635 & 1.128 .009 & 1.154 .569 & 6,3 \\
\hline
\end{tabular}

FUENTE: DGT. Boletín Estadistico General. Elaboración propia.

Tal y como avanzábamos, la TABLA No 4 permite apreciar de modo claro la distribución de los niveles de motorización regional para cada una de las cuatro provincias gallegas según la naturaleza del parque. Como bien se advierte, son las dos provincias atlánticas las que disfrutan de los mayores niveles de motorización, mereciendo una atención especial el parque de turismos y de vehículos pesados; el índice de ambos parques llega a duplicar el alcanzado por Lugo y Ourense.

La importancia de los niveles de motorización regional dentro de la operatividad de los transportes es doble. En primer lugar, habría que consignar que esta variable explica en buena medida el valor potencial que alcanza la demanda de movilidad intrarregional o interregional, según sean sus extremos de viaje. Al tiempo, las demandas de transporte consignadas en las IMD responden tácitamente a una explícita motivación del viaje, lo que permite concluir que en unos itinerarios se alcancen mayores niveles de tráfico que en otros. Entonces, resulta fácilmente deducible consignar que la localización de las principales áreas generadoras o 
captoras del tráfico regional serán aquellas que registren los mayores niveles de motorización y por tanto también, las mayores demandas de transporte. Además, es la propia motivación del viaje la que acaba por determinar la participación diferenciada del parque móvil, según sea la naturaleza del mismo (vehículos ligeros y pesados). Por tanto, los niveles alcanzados por la demanda de movilidad habrán de estudiarse en base a la interacción de cuatro factores: la localización espacial de las áreas extremo de viaje, el itinerario de la red regional que registra los mayores índices de movilidad diaria (IMD), la naturaleza del parque demandante de tráfico (pesado o ligero) y, finalmente, la distribución horaria de los tráficos. La conjunción de tales indicadores nos permite refrendar que el tipo de tráfico que opera en la red regional deberá beneficiarse de la oferta de una adecuada conectividad territorial. Así pues, el papel que se le asigna a la oferta de conectividad territorial no es otro que el hecho de potenciar tanto la generación como la captación de unos flujos de tráfico intensos, crecientes y a la vez fluídos. Se trata de garantizar, mediante la inversión pública en conectividad territorial, la plena igualdad de oportunidades en el momento canalizar su potencialidad productiva hacia unos mercados cada vez más selectivos y distantes; pero, en todo momento, los tipos de tráfico deberán ser acordes al modelo de desarrollo regional a potenciar en Galicia. En conclusión, los niveles de transporte con O-D Galicia y las demás regiones, concluye por mostrarnos como la consecución de unos estándares de baja accesibilidad territorial marginarán el desarrollo económico de la Comunidad Autónoma gallega.

Tabla $\mathbf{n}^{0}$ 4. Distribución provincial del parque regional de vehículos. 1994.

\begin{tabular}{lrrrrrrr}
\hline (1) & \multicolumn{1}{c}{ CO } & \multicolumn{1}{c}{ LU } & \multicolumn{1}{c}{ OU } & \multicolumn{1}{c}{ PO } & GA & \multicolumn{1}{c}{ ES } & \multicolumn{1}{c}{ \%G/E } \\
\hline A & 354.771 & 121.100 & 128.625 & 319.136 & 923.632 & 13.733 .794 & 6,7 \\
B & 90.834 & 41.962 & 44.089 & 84.019 & 260.904 & 4.215 .385 & 6,1 \\
C & 1.766 & 587 & 555 & 1.365 & 4.273 & 47.088 & 9,1 \\
D & 23.494 & 11.076 & 8.604 & 27.654 & 70.828 & 1.612 .295 & 4,3 \\
\hline
\end{tabular}

(1) Tipos de vehículo = A, turismos; B, camiones y furgonetas; C, autobuses; D, otros. CO, A Coruña; LU, Lugo; OU, Ourense; PO, Pontevedra; GA, Galicia; ES, España.

FUENTE: Elaboración propia a partir del Boletín Informativo y el Anuario Estadístico General del $D G T$.

Ciertamente, las inversiones en infraestructuras de transportes y comunicaciones propuestas por los respectivos planeamientos de la Administración Central y Autonómica tendían, en ambos casos, a incrementar los niveles de accesibilidad territorial de Galicia. En estos justos términos, el propósito perseguido no era otro que lograr una plena articulación territorial que contrarrestase los adversos efectos que le conferían a Galicia la condición de periferia económica. En base a todo lo referido, las interacciones regionales en las que uno de sus extremos de viaje estaba localizado en Galicia y el otro bien en la Meseta o en la fachada cantábrica, han experimentado considerables aumentos en los niveles de accesibilidad. Como se constata en las TABLAS $\mathrm{N}^{\circ} 5 \mathrm{y} \mathrm{N}^{\circ} 6$, la integración regional de Galicia registra crecimientos notorios; sin embargo, las interacciones con la fachada cantábrica 
todavía no alcanzan unos niveles aceptables por no existir una autovía que enlace todas las Comunidades Autónomas allí radicadas.

Tabla $\mathbf{n}^{0}$ 5. La accesibilidad interterritorial de Galicia con la Meseta. 19841993.

\begin{tabular}{llll}
\hline \multicolumn{1}{c}{ Itinerario } & A & B & C \\
\hline Madrid-A Coruña & $7 \mathrm{~h} .30 \mathrm{~m}$. & $7 \mathrm{~h} .00 \mathrm{~m}$. & $30 \mathrm{~m}$. \\
Madrid-Pontevedra. & $7 \mathrm{~h} .40 \mathrm{~m}$. & $7 \mathrm{~h} .20 \mathrm{~m}$. & $20 \mathrm{~m}$. \\
\hline Valladolid-A Coruña. & $5 \mathrm{~h} .50 \mathrm{~m}$. & $5 \mathrm{~h} .30 \mathrm{~m}$. & $20 \mathrm{~m}$. \\
Valladolid-Pontevedra & $6 \mathrm{~h} .00 \mathrm{~m}$. & $5 \mathrm{~h} .30 \mathrm{~m}$. & $15 \mathrm{~m}$. \\
Valladolid-A Coruña. & 5 h. $50 \mathrm{~m}$. & $5 \mathrm{~h} .30 \mathrm{~m}$. & $20 \mathrm{~m}$. \\
\hline A.
\end{tabular}

$A$, tiempo de viaje en 1984; $B$, tiempo de viaje en 1993; $C$, diferencial de accesibilidad.

FUENTE: Elab. Propia a partir del Plan General de Carreteras. Balance.

Tabla $\mathbf{n}^{0}$ 6. La accesibilidad interterritorial de Galicia con la fachada cantábrica. 1984-1993.

\begin{tabular}{lllc}
\hline \multicolumn{1}{c}{ Itinerario } & \multicolumn{1}{c}{ A } & B & C \\
\hline Bilbao-A Coruña & $10 \mathrm{~h} .00 \mathrm{~m}$ & $8 \mathrm{~h} .40 \mathrm{~m}$ & $1 \mathrm{~h} .20 \mathrm{~m}$ \\
\hline Santander-A Coruña & $8 \mathrm{~h} .26 \mathrm{~m}$ & $7 \mathrm{~h} .25 \mathrm{~m}$ & $1 \mathrm{~h} .01 \mathrm{~m}$ \\
\hline Oviedo-A Coruña & $5 \mathrm{~h} .17 \mathrm{~m}$ & $4 \mathrm{~h} .38 \mathrm{~m}$ & $0 \mathrm{~h} .39 \mathrm{~m}$ \\
\hline
\end{tabular}

$A$, tiempo de viaje en 1984; $B$, tiempo de viaje en 1993; $C$, diferencial de accesibilidad.

FuENTE: Elaboración Propia a partir del Plan General de Carreteras. Balance.

Para finalizar el análisis de los niveles de accesibilidad territorial, nos detendremos un instante en los registros intraterritoriales. A la luz de las informaciones recogidas en la $\mathrm{TABLA} \mathrm{N}^{\circ}$ 7, puede advertirse como la accesibilidad intrarregional para los principales centros urbanos de Galicia ha conocido un considerable incremento en las velocidades comerciales, lo que conlleva un notorio descenso en los tiempos de viaje para estas interrelaciones urbanas. Resulta del todo obvio constatar que el incremento de los umbrales de accesibilidad interna ha venido propiciados por la creación de una importante red de comunicaciones de alta capacidad de carga, cuya titularidad corresponde a la Administración del Estado y a la Autonómica. De una parte, la incidencia de las ejecuciones del programa de autovías del Estado ha puesto de relieve como estas infraestructuras de transporte permiten al territorio regional la consecución de dos ventajas comparativas más. La primera sería aquella que abundaría en un mayor grado de vertebración y cohesión territorial. En relación con lo anterior, estos ejes refuerzan la conectividad territorial interna de Galicia e impulsan las relaciones de transporte entre áreas más distantes.

Por último, tan sólo resta referir que los niveles de accesibilidad intraterritorial alcanzados por parte de las cabeceras de comarca no se pueden equiparar, salvo 
en casos puntuales, con los estándares de accesibilidad de los principales centros urbanos regionales. Este hecho bien puede ser explicado en base a la propia malla de comunicaciones, la cual muestra una jerarquización de la red regional que beneficia a los centros poblacionales de mayor rango-tamaño de Galicia. Ahora bien, las mejoras que progresivamente se fueron llevando a cabo dentro de la nueva política autonómica de transportes tratan de potenciar la consecución de una red que supere el aislamiento interno de Galicia. De ello cabe deducir que las principales actuaciones programadas en materia de dotación de infraestructuras de transporte se han orientado hacia la vertebración de Galicia, para así contrarrestar los desequilibrios regionales internos.

Tabla $\mathbf{n}^{0}$ 7. La accesibilidad intraterritorial regional de los principales centros urbanos regionales respecto A Coruña.

\begin{tabular}{lr}
\hline A Coruña-Ferrol & $0: 46 \mathrm{~h}$ \\
A Coruña-Santiago & $0: 45 \mathrm{~h}$ \\
\hline A Coruña-Pontevedra & $1: 15 \mathrm{~h}$. \\
A Coruña-Vigo & $1: 28 \mathrm{~h}$ \\
\hline A Coruña-Ourense & $2: 14 \mathrm{~h}$ \\
A Coruña-Lugo & $1: 00 \mathrm{~h}$ \\
\hline
\end{tabular}

FUENTE: Elaboración propia.

Con atención a la conectividad territorial efectuada mediante la oferta de transporte multimodal, merece la pena reiterar que Galicia tampoco disfrutaba de buenos indicadores a este respecto. Para comenzar, hay que aludir a que uno de los principales factores diferenciales del territorio regional gallego frente al resto del Estado, reside en la fuerte dispersión de su hábitat. Galicia dispone aproximadamente de la mitad de los asentamientos del territorio nacional (TABLA $\mathrm{N}^{\circ} 8$ ) y, por consiguiente, esta tupida trama de asentamientos demanda perentoriamente una oferta de conectividad que posibilite la articulación del territorio a las escalas parroquial, comarcal, provincial, regional e interregional.

Tabla no 8. Distribución provincial de las entidades de población en Galicia. 1997.

\begin{tabular}{rrrrr}
\hline A Coruña & Lugo & Ourense & Pontevedra & Galicia \\
\hline 10.242 & 9.161 & 3.688 & 6.088 & 29.1798 \\
\hline
\end{tabular}

FUENTE: INE. Elaboración propia.

Así pues, la intensa ocupación del territorio gallego señala como son los centros poblacionales menores de 1.000 habitantes los núcleos que están por encima del 52,6\% del hábitat gallego. Sin embargo, no concluye ahí la particularidad del hábitat regional. Al afinar un poco más el comentario apreciamos como el porcen- 
taje de las entidades de población de menos de 100 habitantes alcanzan unos niveles de participación del orden del $87,9 \%$. Asimismo, al examinar con detenimiento la participación que han seguido los diferentes núcleos poblacionales desde el Censo de 1950 hasta el de 1991, apreciamos como son los núcleos de menos de 1.000 habitantes los que concentran los mayores porcentajes de población gallega. En contraposición a este singular fenómeno dentro del conjunto del Estado, la tendencia futura, como bien se advierte en la TABLA $\mathrm{N}^{\circ} 9$, tiende a cambiar. En efecto, a tenor de la participación de cada segmento del hábitat gallego apreciamos como son los núcleos de más de 20.000 habitantes los que van a experimentar un mayor nivel de crecimiento, en virtud a la fase de concentración poblacional seguida durante las décadas de los años 60 a 90. Aún más, al examinar los niveles de crecimiento municipal de Galicia apreciamos como es en la periferia de las principales ciudades atlánticas donde se localizan los mayores porcentajes de crecimiento relativo de la población. Este fenómeno viene a suponer, desde el punto de vista del desigual crecimiento poblacional que conocen las ciudades centrales y sus periferias, la irradiación del crecimiento; fenómeno éste que en los casos de A Coruña y Vigo adquiere plenamente el rango de metropolitanización. La difusión del crecimiento poblacional se caracteriza por partir de los lugares centrales y dirigirse hacia aquellos espacios de la periferia mejor dotados de equipamientos públicos e infraestructuras de transporte, por lo que sus índices de accesibilidad respecto de la ciudad central son elevados.

Tabla no 9. Distribución de la población gallega de hecho según el tamaño de los asentamientos para 1950,1970 y 1991.

\begin{tabular}{lrrrrrr}
\hline $\begin{array}{c}\text { Asentamientos } \\
\text { (población) }\end{array}$ & $\mathbf{1 9 5 0}$ & & $\mathbf{1 9 7 0}$ & $\mathbf{1 9 9 1}$ \\
\hline & Población & \% & Población & \% & Población & \% \\
\hline$<1.000$ & 2.009 .974 & 77,2 & 1.726 .330 & 66,2 & 1.431 .426 & 52,6 \\
$1.000-5.000$ & 189.248 & 7,3 & 230.900 & 9,5 & 265.057 & 9,7 \\
\hline $5.000-20.000$ & 43.407 & 1,7 & 56.298 & 2,2 & 178.945 & 6,6 \\
$>20.000$ & 361.571 & 13,9 & 570.146 & 22,1 & 845.017 & 31,0 \\
\hline
\end{tabular}

FUENTE: Elaboración propia a partir de los censos del INE.

Sin afán de extendernos sustancialmente sobre este epígrafe, referiremos que la estructura poblacional gallega sitúa en su orden jerárquico superior a las Áreas Metropolitanas de A Coruña y Vigo. En un nivel inmediato al anterior se incluirían las demás ciudades del territorio regional; para, posteriormente, sucederles en importancia los núcleos urbanos que desempeñarían las funciones de cabeceras de comarca. Por último, y cerrando piramidalmente la trama de los asentamientos regionales, figuraría la aldea en su más pura expresión, que en Galicia presenta caracteres bien singulares en lo referente a total de población y estructura (PAZO LABRADOR, 1995).

Al tratar de examinar la correlación existente entre la oferta de conectividad y el peso de la población gallega, es necesario someter a examen las informaciones 
correspondientes a la TABLA N 10 , las cuales están referidas a la titularidad jurídica de la red viaria regional según su distribución provincial. En ella descubrimos dos hechos que prevalecen por encima de los restantes. El primero lo constituye la desigual distribución provincial de la red regional de comunicaciones según su titularidad jurídica. Los destacados niveles que alcanza la red de las diputaciones frente a la de otros niveles de la Administración nos traduce que una buena parte de la red regional disfruta de una capacidad de carga relativamente baja. Por ello, este fenómeno confirma, con suma nitidez, como el índice de dispersión geográfica del hábitat regional, al que hacíamos mención más arriba, repercute en la creciente demanda de conectividad territorial.

Tabla $\mathbf{n}^{0}$ 10.Distribución provincial de la red de carreteras regional según la titularidad jurídica de la red para 1994.

\begin{tabular}{crrrrrrr}
\hline Red & CO & LU & OU & PO & GA & ES & \%G/E \\
\hline A & 408 & 572 & 373 & 480 & 1.833 & 22.536 & 8,1 \\
B & 1.592 & 1.305 & 988 & 1.109 & 4.994 & 72.565 & 6,8 \\
C & 2.120 & 4.058 & 1.849 & 1.765 & 9.792 & 67.095 & 14,5 \\
\hline D & 4.120 & 5.935 & 3.210 & 3.354 & 16.619 & 162.196 & 10,2 \\
\hline E & - & - & - & - & 15.397 & 168.470 & 9,1 \\
\hline
\end{tabular}

A, Rige; $B$, Red autonómica; $C$, Red de las Diputaciones y Cabildos; D, total; E, otras. CO, A Coruña; LU, Lugo; OU, Ourense; PO, Pontevedra; GA, Galicia; ES, España FUENTE: MOPTMA. Anuario Estadístico. Elaboración Propia.

En segundo término, al examinar la participación porcentual de la red regional según su titularidad jurídica frente al total de España, descubrimos que los umbrales obtenidos por la misma se sitúan siempre por encima de la participación demográfica de Galicia dentro del conjunto del Estado. Efectivamente, la participación de la población gallega dentro de la total española está cifrada, en el Censo de 1991, en un 6,89\%, mientras que la participación de la red viaria regional, según la propia titularidad jurídica, excede siempre de este nivel.

No obstante, esta realidad encubre diferencias sustanciales en lo que se refiere a la operatividad de la red. En efecto, si tratamos de analizar la participación de la calificada como de alta capacidad o, de un modo más concluyente, si analizamos las participaciones de la red según el tipo de firme y ancho de la calzada, podemos comprobar como Galicia está bien lejos de equipararse con aquellas regiones más desarrolladas. Esto último adquiere su máxima expresión cuando observamos en la TABLA No 11 como en Galicia todavía continúa operativa una importante longitud de red con firme de macadam. La importancia de este factor es doble. Por un lado cabe referir que las velocidades comerciales asociadas a este material son bajas. Por otro, la localización territorial de esta red nos señala como son las provincias del interior las que poseen la práctica totalidad de este tipo de red. Para concluir, merece ser apuntado, que Galicia participaba de niveles bajos en la dotación de red de alta capacidad (TABLA $\mathrm{N}^{\circ} 12$ ); en especial, de aquella que tiene por objeto ofrecer los mayores niveles de cobertura al territorio regional. Así dicho, 
ésta no puede ser otra más que la que se destina a cubrir los itinerarios interurbanos. Pero también, resulta de todo orden cierto que la tendencia futura es hacia el crecimiento de la red regional de alta capacidad, una vez se ejecuten los tramos programados de la red nacional de autovías y los contemplados en la programación del Plan de Estradas de Galicia 1990-2000 y su ampliación para el período 1996-2006.

Tabla $\mathbf{n}^{0}$ 11.Distribución provincial de la red regional de carreteras según el pavimento de su firme. 1994.

\begin{tabular}{crrrrrrr}
\hline Firme & CO & \multicolumn{1}{c}{ LU } & OU & PO & GA & ES & \%G/E \\
\hline Red viaria regional de una calzada & \multicolumn{5}{c}{} \\
\hline $1 A$ & 1 & 474 & 48 & -- & 523 & 3.532 & 14,8 \\
$1 B$ & 1.436 & 959 & 821 & 2.141 & 5.357 & 71.258 & 7,5 \\
$1 C$ & 2.538 & 4.500 & 2.331 & 1.105 & 10.474 & 79.658 & 13,1 \\
$1 D$ & 3.975 & 5.933 & 3.200 & 3.246 & 16.354 & 154.448 & 10,6 \\
\hline \multicolumn{6}{l}{ Red viaria regional de doble calzada (autovias } & y autopista) & \\
\hline $2 A$ & 145 & 2 & 10 & 108 & 265 & 7.748 & 3,4 \\
$2 B$ & 4.120 & 5.935 & 3.210 & 3.354 & 16.619 & 162.196 & 10,2 \\
\hline
\end{tabular}

1A, Macadam y otros; $1 B$, Hormigón o Aglomerado asfáltico; 1C, Tratamiento superficial;1D, Total; $2 A$, Hormigón o aglomerado asfáltico; $2 B$, Total red viaria regional. CO, A Coruña; LU, Lugo; OU, Ourense; PO, Pontevedra; GA, Galicia; ES, España. FUENTE: MopTMA. Anuario Estadístico. Elaboración Propia.

Volviendo sobre nuestros pasos y retomando la importancia que a Galicia le supone la dotación de una conectividad territorial adecuada a una sociedad que camina hacia cotas elevadas de bienestar social, mencionaremos que este objetivo únicamente será posible si las políticas de desarrollo regional inciden en acciones estratégicas tendentes a impulsar de manera equitativa la igualdad de oportunidades respecto de otros territorios del Estado, tal y como contempla el Plan de Desarrollo Regional Galicia 1994-1999. En este contexto, merece ser destacada la participación que cumple en la Ordenación del Territorio (O.T.) la planificación de las infraestructuras de transporte y comunicaciones; las cuales, son una condición indispensable pero insuficiente en sí misma si no va acompañada de otras políticas de acción que tiendan a dinamizar la estructura socioproductiva de Galicia.

La planificación de las comunicaciones gallegas ha atravesado por fases bien diferenciadas. Por tal motivo, no es un hecho casual que la auténtica transformación de la trama regional sea un acontecimiento relativamente reciente y enmarcado en el contexto de la España de las Autonomías (RODRÍGUEZ SÁEZ, L, 1980), dejándose sentir con mayor incidencia desde finales de los años 1980 y comienzos de los 1990. Por el contrario, desde la segunda mitad de los 70 hasta la primera de los 80, dos fenómenos explicaban el estatismo. De una parte, la política desarrollo regional respondía a un canon centralista de la Administración. Posteriormente, Galicia entra en una fase de transición caracterizada por la inoperancia y la caren- 
cia de vinculación entre lo programado y lo ejecutado. Esta fase constituye la antesala de la aprobación del Estatuto de Autonomía de Galicia mediante la Ley Orgánica de 1/1981 de 6 de Abril, a través del cual se dota al territorio de las cuatro provincias de las competencias de ordenación del territorio con un desarrollo íntegro en Galicia siempre que no sean de interés general del Estado.

Tabla $\mathbf{n}^{0}$ 12. Distribución provincial de la red viaria regional según la capacidad y ancho de la calzada. 1994.

\begin{tabular}{lrrrrrrr}
\hline & CO & LU & OU & PO & GA & ES & \%G/E \\
\hline Ancho & & & & & & & \\
\hline$<5 \mathrm{~m}$. & 1.302 & 3.686 & 1.473 & 1.030 & 7.491 & 40.484 & 18,5 \\
$5-7 \mathrm{~m}$. & 1.332 & 1.394 & 891 & 1.426 & 5.043 & 73.264 & 6,9 \\
$>7 \mathrm{~m}$. & 1.341 & 853 & 836 & 790 & 3.820 & 40.700 & 9,4 \\
\hline Total & 3.975 & 5.933 & 3.200 & 3.246 & 16.354 & 154.448 & 10,6 \\
\hline Capacis
\end{tabular}

Capacidad

\begin{tabular}{lrrrrrrr}
\hline Doble calzada & 24 & 2 & 9 & 18 & 53 & 1.251 & 4,2 \\
Autov.-autop. libres & 26 & - & 1 & 29 & 56 & 4.474 & 1,3 \\
Autop. de peaje & 95 & - & - & 61 & 156 & 2.023 & 7,7 \\
\hline Total absoluto & 4.120 & 5.935 & 3.210 & 3.354 & 16.619 & 162.196 & 10,2 \\
\hline CO, A Coruña; LU, Lugo; OU, Ourense; PO, Pontevedra; GA, Galicia; ES, España. \\
FUENTE: MOPTMA. Anuario Estadistico.
\end{tabular}

\section{LAS INTERACCIONES INTERMODALES DE LA DEMANDA DE MOVILIDAD REGIONAL EN GALICIA.}

El análisis de la movilidad regional según la modalidad del parque y la naturaleza de los desplazamientos (viajeros o mercancías), revela dos elementos de considerable interés. El primero es aquel que se refiere a las principales interacciones de Galicia con los demás territorios regionales y que se materializan a través de los ejes de acceso a la Meseta: N-VI, N-550, N-120 y las autovías del Noroeste y de As Rías Baixas; de este modo se da cobertura a la demanda de transporte radial. Por otra parte, las demandas de transporte transversal tienden a cubrirse por medio del corredor transcantábrico. Las interacciones regionales de Galicia respecto de las ciudades del interior de España son cubiertas por las dos autovías mencionadas, juntamente con el trazado de la N-VI para el tráfico interurbano. En virtud de tal condición, la oferta de una conectividad territorial dotada de una alta capacidad de carga y unos estándares de accesibilidad importantes contribuyen a explicar como progresivamente Galicia tiende a convertirse en una Comunidad Autónoma que presenta notorias ventajas comparativas. Éstas le vienen dadas por su condición de ser una puerta abierta al mar para todas aquellas demandas de transporte generadas o atraídas en las provincias de: Castilla-León, y, sobre todo, Madrid.

En lo tocante a las demandas de transporte transversal, el corredor transcantábrico no se encuentra en una posición de menor interés respecto al anterior. Las elevadas potencialidades que ofrece la conectividad regional transcantábrica tanto 
en lo que concierne a las interacciones regionales como a los propios flujos transnacionales, sirven para integrar plenamente a Galicia en el conjunto de la U.E. por propiciarle el acceso a las redes transeuropeas vía Irún. Así pues, este corredor está llamado a ser uno de los ejes de transporte que en fechas futuras ostentará los mayores niveles de crecimiento del transporte, por cuanto integra a un total de cinco Comunidades Autónomas (País Vasco, Cantabria, Asturias, Galicia y Navarra) dotadas de una estructura productiva muy competitiva en el acceso a los mercados nacionales e internacionales. Las espectativas de crecimiento del tráfico sobre los territorios por los que discurre el corredor transcantábrico son claras, toda vez que en ellos existen unos notables índices de motorización regional. Más aún, dentro de la importancia que el índice de motorización alcanza en las Comunidades Autónomas mencionadas es de destacar la importancia del parque de vehículos pesados, por cuanto son éstos los destinados a cubrir los transportes de mercancías y, por consiguiente también, es este tipo de tráfico el que presenta unas demandas de movilidad de mayor radio de acción. Sin ánimo de detener el comentario en el interés que estos dos corredores le confieren a la demanda de transporte regional, cabe enfatizar el interés que para Galicia tiene un tercero o, por qué no decirlo, incluso un cuarto.

Efectivamente, en el contexto del Estado de las Autonomías, consecuencia de un nuevo marco de organización territorial del Estado, cabe destacar el papel que cada autonomía deberá cumplir ante hechos tan relevantes como la integración territorial del desarrollo regional. Así, los nuevos derroteros de la Ordenación del Territorio explican como las políticas de inversión pública en materia de transportes y comunicaciones tratan de paliar los efectos perniciosos de una excesiva dependencia de los flujos radiales, dado que este tipo de transportes obran en detrimento de la accesibilidad del transporte regional gallego. En suma, este adverso factor -la radialidad de los tráficos- merece ser corregido con prontitud, por parte de los territorios que en mayor medida están padeciendo este lastre. Consecuentemente, resulta del enorme interés emprender la ejecución de itinerarios transversales que reporten a las matrices $\mathrm{O}-\mathrm{D}$ un sustancioso refuerzo a las interacciones regionales al incrementar la accesibilidad territorial y la velocidad comercial que condicionan a la baja los tiempos de viaje; pero al mismo tiempo también, logran captar o generar nuevas motivaciones de transporte.

En este orden de cosas, el corredor que mejor tiende a facilitar la integración transversal de las comunicaciones para Galicia sería el eje subcantábrico, por ser éste el que posibilita los enlaces de Galicia con el corredor del Ebro, Cataluña y Comunidad Valenciana, mediante previo paso por la Comunidad de CastillaLeón. Además, el cuarto de los ejes viarios necesitado de una fuerte implementación, es el destinado a la consecución de unas intensas relaciones entre Galicia y Portugal. Ciertamente, Galicia debe apostar en firme por las relaciones con el norte de Portugal para generar un importante eje de desarrollo económico (Eje Atlántico) que capte crecientes inversiones públicas y, por extensión de lo anterior, refuerce los flujos de transporte entre el sistema de ciudades y comarcas de Galicia y sus homólogas portuguesas.

En las TABLAS N $\mathrm{N}^{\circ} 13$ y N $^{\circ} 14$ podemos apreciar la participación de los niveles de tráfico de cada modo de transporte entre los territorios que hemos señalado. 
Como se advierte, el peso de la carretera tanto en el transporte de viajeros como de mercancías es considerable para casi todas las interacciones regionales. Ahora bien, en la fachada cantábrica merece ser destacado el importante papel que cumple el transporte de mercancías por vía marítima en los puertos de las Comunidades Autónomas allí localizadas.

Tabla $\mathbf{n}^{0}$ 13. Reparto modal en las principales relaciones de viajeros y mercancías entre Galicia y la Fachada Cantábrica. 1988.

\begin{tabular}{lrrrrrrrr}
\hline $\begin{array}{l}\text { Relación territo- } \\
\text { rial }\end{array}$ & \multicolumn{3}{c}{ Viajeros } & \multicolumn{5}{c}{ Mercancías } \\
\hline & TO & \%CA & \%FE & \%AV & TO & \%CA & \%FE & \%BA \\
\hline País Vasco-Galicia & 1.285 & 60,1 & 31,4 & 8,5 & 2.721 & 29,4 & 8,0 & 62,6 \\
Cantabria-Galicia & 413 & 95,4 & 4,6 & - & 833 & 32,8 & 14,6 & 52,6 \\
Asturias-Galicia & 1.922 & 92,6 & 5,7 & 1,7 & 7.424 & 46 & 4,4 & 49,6 \\
\hline
\end{tabular}

TO, total; CA, carreteras; FE, ferrocarril; AV, avión.

FUENTE: Plan General de Carreteras. Balance, 1994. Elaboración Propia.

Tabla $\mathbf{n}^{0}$ 14. Reparto modal en las principales relaciones de viajeros y mercancías entre Galicia y la Meseta. 1988.

\begin{tabular}{lrrrrrrrr}
\hline $\begin{array}{l}\text { Relación territo- } \\
\text { rial }\end{array}$ & \multicolumn{3}{c}{ Viajeros } & & \multicolumn{4}{c}{ Mercancías } \\
\hline & TO & \%CA & \%FE & \%AV & TO & \%CA & \%FE & \%BA \\
\hline Madrid-Galicia-Ee & 1.276 & 70,5 & 29,5 & - & 977 & 99,3 & 0,7 & - \\
Madrid-Galicia $W$ & 4.510 & 37,4 & 31,4 & 31,2 & 2.119 & 69,9 & 30,1 & - \\
León/Vall.-Galicia & 1.742 & 82,3 & 16,9 & 0,8 & 4.192 & 59,4 & 40,6 & - \\
\hline
\end{tabular}

TO, total; CA, carreteras; FE, ferrocarril; AV, avión.

FuENTE: Plan General de Carreteras. Balance, 1994. Elaboración Propia.

Para no cerrar en falso las reflexiones que nos suscita este apartado, realizaremos a modo de conclusión de todo lo anterior un apunte más. De la TABLA No 14 referida a los tráficos de Galicia con la Meseta según la modalidad de los tráficos, se obtienen dos conclusiones que permiten aventurar la presencia de una oferta de transporte nada adaptada y especializada a sus propias potencialidades. Comenzando por el principio, la carretera es el modo que predomina por encima de todos los demás en lo que a niveles de tráfico respecta y sin distinción alguna en lo tocante a la mera naturaleza del tráfico (mercancías o viajeros). Ahora bien, cuando tratamos de analizar los niveles alcanzados por el ferrocarril para estas mismas relaciones interregionales, descubrimos como éste participa de unos niveles escasos, lo que permite constatar una clara inadaptación de este sistema a la demanda, así como una desfavorable competencia de este sistema respecto de los demás. Por otra parte, se advierte como la demanda de movilidad progresivamente es más exigente respecto de la accesibilidad. Es en función de este hecho por el que se explica como el transporte aéreo está captando crecientes niveles de tráfico entre Galicia y la Meseta; sustentado, además, por una liberalización del mercado. Este 
contexto ha sido el que ha propiciado la libre concurrencia de operadores del transporte aéreo, lo cual se ha visto traducido en una flexibilización tarifaria que está revirtiendo en la captación de ingentes niveles de demanda por parte de los tres aeropuertos gallegos.

Finalmente resta dejar constancia que para alcanzar una oferta de transportes equilibrada y adaptada a las pautas que impone el modelo de desarrollo regional, es preciso corregir la acentuada competitividad entre modos. Para proceder a dar cumplida respuesta a tal propósito, es menester proponer un Plan Intermodal de Transportes que posibilite la eficaz especialización de cada sistema de transporte a los propios caracteres de la demanda de viaje y según sea y se localice la misma.

\section{BIBLIOGRAFÍA.}

- ARESPaCochaga y Felipe, J. (1967): Galicia, esa península, Edit. Ateneo. Madrid.

- CAMBRE MARIÑO, J. (1968): Galicia ante el desequilibrio regional de España. Edit. Galaxia. Colección Agra Aberta, 1. Vigo.

- CотоP (1993): Plan de Estradas de Galicia 1990-2000. Xunta de Galicia. Santiago.

- DÍAZ FERNÁNDEZ, J. A. (1997): El sistema de transporte y comunicaciones en el A.M. de A Coruña. 1970-1991. Universidad de Santiago.

- GÓMEZ BARAHONA, A. (1991): La Planificación Económica Regional: Análisis Jurídico. Edit. Ministerio de Economía y Hacienda Colección Estudios Jurídicos. Instituto de Estudios Fiscales. Madrid.

- MARCO BORDETAS, L. ET. AL. (1977): Incidencia del transporte en el desarrollo regional. Subsecretaría de Planificación. Presidencia del Gobierno. Madrid.

- INE (varios años): Censos de población y viviendas. Madrid.

- MoptMa (1993): Plan General de Carreteras. Balance. Madrid.

- MoptMa (1994): Plan Director de Infraestructuras, 1993-2007. 2a Edic. Madrid.

- PAZO LABRADOR, J. (1995): La trama de los asentamientos rurales en las rías bajas gallegas. Diputación provincial de Pontevedra.

- PASTOR ANTOLÍN (1986): L. La red de transporte en Castilla y León. Edit. Ámbito. Valladolid.

- Pena TRAPero, X. B. (1974): Galicia e os plans de desenvolvemento, Edit. Sep. Pontevedra.

- Pena Trapero, X. B. (1972): A Planificación rexional e Galicia. Edit. Sep. Pontevedra.

- PÉREZ TOURIÑO, E. (Coord.) (1997): Infraestructuras y Desarrollo Regional: Efectos Económicos de la Autopista del Atlántico, Edit. Civitas, ENA e IDEGA. Madrid.

- Plan de Desarrollo Regional de Galicia (PDR) 1989-1993. PDR Galicia 1994-1999. Santiago.

- RICHARDSON, H. W. (1971): «Desarrollo regional y urbano en España». Revista española de economía, núm. 1, III, Enero-Abril. Madrid.

- Richardson, H. W. Política y Planificación del Desarrollo Regional en 
España. Edit. Alianza Universal. Madrid. 1976.

- RodríGUEz SÁEZ, L. (1980): «Política de Transportes», en GÁMIR CASARES, L. Política económica de España. 2. Autonomías, sectores, objetivos. Edit. Alianza. Madrid.

- SEQUEIROS TIZÓN, X. G. (1979): La polarización espacial en el crecimiento económico español. El caso gallego. Universidad de Santiago de Compostela.

- Sequeiros Tizón, X. (1986): El desarrollo económico en Galicia I. Agricultura y Mercado Interior. Edit. Universidad de Santiago.

- TABOADA ARCEO, A. (1969): Galicia dormida ante el II Plan Económico y Social. Proposición abierta a quienes puedan y deben redimir a Galicia de su pobreza. Edit. Cámara Oficial de Comercio Industria y Navegación de A Coruña.

- TAMAMES, R. (1980): Estructura económica de España. Edit. Alianza. Madrid.

RESUMEN: Anclados en el contexto del Estado de las Autonomías, la dotación de inversión pública en materia de infraestructuras productivas básicas de transportes y comunicaciones contribuye de manera bien representativa a velar por el principio de la $\mathrm{co}$ hesión económica y social del Estado. Así pues, dos son las externalidades positivas más destacadas con las que cumple identificar al esfuerzo inversor señalado. En un extremo figuraría la dotación de conectividad territorial destinada a impulsar la articulación territorial de la CC.AA. de Galicia respecto de los demás restantes territorios regionales. El segundo de los oportunamente mencionados sería, en rigor, la dotación de accesibilidad territorial con la que comúnmente se identifica al esfuerzo presupuestario ejecutado. Con todo, pues, es la accesibilidad territorial resultante uno de los factores que en último extremo contribuyen a explicar la aparición de fenómenos tan relevantes para el desarrollo regional como ciertamente es el de crowdin-in.

PALABRAS CLAVE: Política económica de desarrollo regional, periferia económica, accesibilidad territorial, conectividad territorial, efecto barrera o conectividad territorial.

ABSTRACT: Anchored in the context of the State of the Autonomies, the endowment of public investment as regards basic productive infrastructures of transports and communications contributes from a very representative way to look after the principle of the economic and social cohesion of the State. Therefore, two are the most outstanding positive advanteges with those that it completes to identify to the effort signal investor. In an end it would figure the endowment of territorial connectivity dedicated to impel the territorial articulation of the DC. AA. of Galicia regarding the other remaining regional territories. The second of those appropriately mentioned ones would be, in rigor, the endowment of territorial accessibility with the one that commonly is identified to the executed budgetary effort. With everything, because, it is the accessibility territorial resultant one of the factors that contribute to explain the appearance of phenomenons so excellent for the regional development in last end as certainly it is that of crowdin - in.

KEY WORDS: The economic policy of regional development, economic periphery, territorial accessibility, territorial connectivity, natural barriers or territorial compartimentalization.

RÉSUMÉ: Ancré dans le contexte de l'État des Autonomies, la dotation d'investissement public comme amitiés les infrastructures productives de base de transports et communica- 
tions contribuent d'une façon très représentative de s'occuper du principe de la cohésion économique et sociale de l'État. Par conséquent, deux sont les advanteges du positif les plus remarquables avec ceux qu'il complète pour identifier à l'investisseur du signal de l'effort. Dans une fin il représenterait la dotation de connectivité territoriale a consacré pour forcer l'articulation territoriale du DC. AA. de Galicia concernant les autres restant territoires régionaux. La seconde de ceux que convenablement mentionnés seraient, dans la rigueur, la dotation d'accessibilité territoriale avec celui qui est identifié à l'effort budgétaire exécuté communément. Avec tout, parce que, c'est l'accessibilité résultant territorial des facteurs qui contribuent pour expliquer l'apparence de phenomenons si excellent pour le développement régional dans dernière fin comme certainement il est cela de crowdin in.

MOTS-CLÉS: La politique économique de développement regional, La périphérie économique, l'accessibilité territoriale, connectivité territoriale, barrières naturelles o compartimentalization territoriaux. 